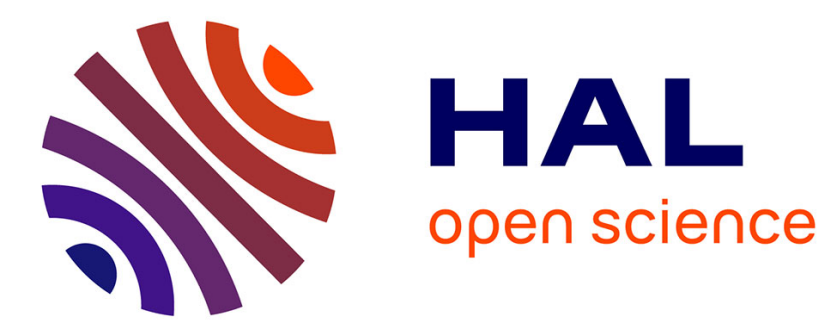

\title{
Chapitre 7 : Eugène Charles Catalan et la théorie des nombres
}

Jenny Boucard

\section{To cite this version:}

Jenny Boucard. Chapitre 7 : Eugène Charles Catalan et la théorie des nombres. Bulletin de la Société des amis de la Bibliothèque et de l'Histoire de l'école polytechnique, 2015, Eugène Catalan (1814-1894, X 1833). Le bicentenaire et le fonds d'archives Catalan-Jongmans, 57, pp.49-56. 10.4000/sabix.1959 . halshs-01351715

\section{HAL Id: halshs-01351715 https://shs.hal.science/halshs-01351715}

Submitted on 10 Apr 2021

HAL is a multi-disciplinary open access archive for the deposit and dissemination of scientific research documents, whether they are published or not. The documents may come from teaching and research institutions in France or abroad, or from public or private research centers.
L'archive ouverte pluridisciplinaire HAL, est destinée au dépôt et à la diffusion de documents scientifiques de niveau recherche, publiés ou non, émanant des établissements d'enseignement et de recherche français ou étrangers, des laboratoires publics ou privés. 


\section{Bulletin de la Sabix}

S Bulletin de la

Société des amis de la Bibliothèque et de l'Histoire de

l'École polytechnique

$57 \mid 2015$

Eugène Catalan (1814-1894, X 1833)

\section{Chapitre 7 : Eugène Charles Catalan et la théorie des nombres}

Jenny Boucard

\section{OpenEdition}

Journals

Édition électronique

URL : http://journals.openedition.org/sabix/1959

DOI : 10.4000/sabix.1959

ISSN : 2114-2130

Éditeur

Société des amis de la bibliothèque et de l'histoire de l'École polytechnique (SABIX)

Édition imprimée

Date de publication : 1 juin 2015

Pagination : 49-56

ISSN : 0989-30-59

Ce document vous est offert par Université de Nantes

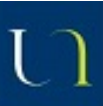

UNIVERSITÉ DE NANTES

Référence électronique

Jenny Boucard, «Chapitre 7 : Eugène Charles Catalan et la théorie des nombres », Bulletin de la Sabix [En ligne], 57 | 2015, mis en ligne le 25 juillet 2018, consulté le 10 avril 2021. URL : http:// journals.openedition.org/sabix/1959; DOI : https://doi.org/10.4000/sabix.1959 


\title{
Chapitre 7
}

\section{EUGÈNE CHARLES CATALAN ET LA THÉORIE DES NOMBRES}

Jenny BOUCARD

Des contributions arithmétiques de Catalan, l'on retient le plus souvent sa célèbre conjecture devenue résultat en 2002 [Voir chapitre 6] ; c'est bien entendu une vision très réductrice de l'œuvre arithmétique de Catalan mais au-delà du résultat en lui-même transparaissent ici certains de ses traits caractéristiques : son goût pour les équations diophantiennes ${ }^{3}$; son habitude d'énoncer ce qu'il appelle lui-même des théorèmes empiriques dont " les démonstrations viendront plus tard ; du moins [il] l'espère » [Catalan 1892, p. 203] ; son intervention, sous diverses formes, dans plusieurs journaux mathématiques ${ }^{4}$ [Illustration III.3].

\author{
VI \\ THÉORĖMES EMPIRIQUES ("') \\ 23. - I. - Ie sextuple de tout nombre impair est la somme de trois \\ carrés. \\ II. - Soit $\mathrm{N}$ ce sextuple. Si $\mathrm{N}-\ddot{3}$ n'est pas carré, $\mathbf{N}$; somme de trois \\ carrés, est aussi la sommẹ de quatre carrés. \\ III. $-\mathrm{n}$ étant un nombre entier, autre que zéro, la quantité $6 \mathrm{n}^{2}+6 \mathrm{n}-3$ \\ èst la somme de trois carrés. \\ IV. - Cette meme quantité est, suivant la grandeur de $\mathrm{n}: 1^{\circ}$ la somme : \\ de six carrés; $2^{\circ}$ la somme de neuf carrés; ...; $\mathrm{n}^{\circ}$ la somme de $6 \mathrm{n}^{2}+6 \mathrm{n}-3$ \\ carrés.
}

Illustration III.3: Théorèmes empiriques de Catalan [Catalan 1892]

Catalan est effectivement reconnu comme un savant très prolifique en termes de publication, qualifié d'" auteur dominant de la presse mathématique " au XIX ${ }^{\mathrm{e}}$ siècle [Verdier 2009a, p. 258-268]. Cela transparaît d'ailleurs dans la liste de 406 publications de Catalan publiée en 1888 dans le troisième volume des Mélanges de mathématiques en 1888, soit six années avant son décès [Catalan 1888, p. 261-272]. Qu'en est-il de sa production arithmétique? Il est toujours délicat de repérer ce qui peut être qualifié de théorie des nombres chez un auteur du XIX ${ }^{\mathrm{e}}$ siècle [Goldstein 1999], que l'on s'appuie sur les définitions, multiples, données par les auteurs eux-mêmes ou sur les classifications proposées dans les journaux dans lesquels Catalan publie, lorsqu'elles existent, ou celles mises en ouvre rétrospectivement dans les journaux mathématiques de recension fondés dans le dernier tiers du XIX ${ }^{\mathrm{e}}$ siècle, comme le Jabrbuch über die Fortschritte der Mathematik. Nous avons relevé plus d'une centaine de références à Catalan dans la compilation History of the Theory of Numbers de Dickson [Dickson 1919-1923], publiée entre 1919 et 1923. À partir du Jahrbuch, qui recense les articles publiés à partir de la fin des années 1860, et de la liste des publications de Catalan évoquée précédemment, nous retrouvons plus d'une soixantaine d'articles pouvant être rattachés à la théorie des nombres ${ }^{5}$. De plus, une partie des contributions de Catalan au domaine de la théorie des nombres n'apparaît pas dans ce relevé parce qu'elles se font sous d'autres formes qu'un article. Nous y reviendrons. Toujours est-il que ces premières données quantitatives suggèrent une activité arithmétique substantielle de la part de Catalan.

\footnotetext{
${ }^{3}$ Une équation diophantienne est une équation polynomiale à coefficients entiers ou rationnels dont la résolution est attendue en nombres entiers ou rationnels.

${ }^{4}$ Rappelons en effet que Catalan énonce cette conjecture dans les Nouvelles annales de mathématiques en 1842, puis la fait paraître en 1844 dans le Journal de Crelle en envoyant une missive à l'éditeur [Catalan 1844a].

${ }^{5}$ Ici, nous ne prenons pas en compte les textes de Catalan abordant des questions de combinatoire qui pourraient être considérés comme relevant de l'arithmétique: par exemple, Catalan y aborde parfois la question de la partition d'un nombre $m$ en $n$ parties [Catalan 1838].
} 
Comme F. Jongmans le souligne, "la vie d'Eugène Catalan s'étend de 1814 à 1894, soit quasiment d'un bout à l'autre du XIX $\mathrm{X}^{\mathrm{e}}$ siècle [...] " ". Nous commençons donc par donner un panorama de la théorie des nombres au XIX ${ }^{e}$ siècle afin de pouvoir situer la production arithmétique de Catalan puis nous nous arrêterons sur trois épisodes ponctuels qui permettent de donner un éclairage sur les pratiques de Catalan mais aussi sur les réseaux d'auteurs dans lesquels il évolue.

\section{La théorie des nombres au XIX ${ }^{\mathrm{e}}$ siècle}

La théorie des nombres est radicalement transformée au cours du XIX siècle [Goldstein \& al. 2007]. Cette période s'ouvre avec la publication de deux traités. Le premier, Essai sur la théorie des nombres de Legendre, est publié en 1798 [Legendre 1798] et offre une synthèse de résultats arithmétiques déjà énoncés et parfois démontrés par Fermat, Euler ou encore Lagrange, souvent centrés sur l'analyse diophantienne. Dans son introduction, Legendre assimile d'ailleurs explicitement la théorie des nombres à l'analyse indéterminée. C'est en 1801 que le second ouvrage, les Disquisitiones arithmeticae de Gauss paraît [Gauss 1801]. Gauss y distingue clairement la théorie des nombres " où l'on considère particulièrement les nombres entiers, quelquefois les fractions, mais où l'on exclut toujours les nombres irrationnels " [Gauss 1801, Préface] et organise le contenu autour d'un nouvel objet arithmétique : les congruences ${ }^{7}$. Les Disquisitiones arithmeticae vont connaître plusieurs formes de réception.

Dans le premier quart du XIX $\mathrm{X}^{\mathrm{e}}$ siècle, c'est la dernière section qui sera très rapidement reprise, tout particulièrement dans des ouvrages d'algèbre: Gauss y propose une méthode de résolution algébrique des équations binômes $x^{n}=1$ ( $n$ premier), fondée sur l'utilisation d'un outil arithmétique, les racines primitives. La théorie des nombres est alors délaissée par la plupart des mathématiciens, au profit de recherches analytiques et/ou liées à des questions astronomiques et physiques.

La situation change progressivement à partir de 1825. Une nouvelle génération de mathématiciens, comprenant par exemple Carl Gustav Jakob Jacobi et Johann Peter Gustav Lejeune-Dirichlet, se familiarise avec l'ouvrage de Gauss au cours de leur formation mathématique, de façon généralement autodidacte. Cela se passe dans le cadre d'un renouveau des mathématiques allemandes, où la théorie des nombres est beaucoup plus soutenue. C'est également à partir de 1825 que sont créés plusieurs journaux mathématiques ou contenant des mathématiques [Verdier 2009b]: même si, à l'exception du Journal de Crelle, leur durée de publication est relativement courte, ils participent à une reconfiguration du paysage éditorial et donnent des possibilités supplémentaires aux jeunes auteurs de faire connaître leurs travaux. Dans le cas qui nous intéresse ici, le nombre d'articles de théorie des nombres publiés augmente sensiblement. Ce phénomène s'accentue à partir de 1835 , lorsque sont lancés les Comptes rendus de l'Académie des sciences de Paris, le Journal de Liouville (1836) ou encore les Nouvelles annales de mathématiques (1842) dans le cas français. Des recherches contemporaines en analyse portant notamment sur les nombres complexes, l'analyse de Fourier ou encore les fonctions elliptiques, sont également mobilisées pour traiter de nombreuses questions arithmétiques, portant sur des thématiques développées par Gauss dans son ouvrage comme les résidus, les lois de réciprocité ou encore la théorie des formes quadratiques. Entre les années 1830 et 1860, un champ de recherche - intitulé analyse algébrique arithmétique [Goldstein \& Schappacher 2007a] - liant des problématiques de théorie des nombres, de la théorie des équations et d'analyse se développe autour d'un réseau international d'auteurs. On retrouve également une caractéristique générale, mais non spécifique ni en opposition avec l'analyse arithmétique algébrique, commune à quasiment toutes les publications arithmétiques françaises dans lesquelles les congruences sont mobilisées: un rapprochement entre équations et congruences, qui induit des questionnements et des méthodes spécifiques [Boucard 2011]. Ainsi, côté français, pratiquement aucune recherche centrée sur les résidus, les lois de réciprocité ou la théorie des formes quadratiques n'est publiée sur cette période.

\footnotetext{
${ }^{6}$ [Jongmans 1996, p. 1]. Dans la suite de l'article, et sauf indication contraire, les indications biographiques sur Catalan sont issues de cet ouvrage.

${ }^{7}$ Deux nombres entiers $a$ et $b$ sont dit congrus modulo un nombre entier $p$ si leur différence est divisible par $p$.
} 
Du point de vue de l'enseignement, les situations allemandes et françaises sont également très différentes: dans l'espace germanique, des manuels centrés sur les congruences - et donc présentant la théorie des nombres comme Gauss dans les Disquisitiones arithmeticae - sont publiés et des cours universitaires sont consacrés à la théorie des nombres. En France, les programmes d'enseignement, plus homogènes et très orientés par les besoins de l'École polytechnique, ne laissent quasiment aucune place à la théorie des nombres. Les quelques points apparaissant progressivement au programme [Belhoste 1995] ne prennent en aucun cas en compte les résultats de Gauss en termes de résidus ou de congruences.

À partir des années 1860, les recherches rattachées à la théorie des nombres se décomposent en plusieurs réseaux d'articles [Goldstein 1999, Goldstein \& Schappacher 2007b]. Ainsi, C. Goldstein et N. Schappacher distinguent trois réseaux principaux : le premier contient des recherches centrées sur des problématiques renvoyant au début du XIX ${ }^{\mathrm{e}}$ siècle (racines primitives, nombres premiers, équation cyclotomique, équations diophantiennes, fractions continues...) dont les auteurs, issus de milieux variés, s'appuient sur les traités de Legendre et Gauss et proclament leur opposition à l'intégration de l'analyse dans la théorie des nombres; le second réseau adopte pour référence le travail analytique de Dirichlet autour des séries, puis intègre les recherches d'analyse complexe de Riemann à la fin du siècle; les auteurs impliqués dans le troisième réseau s'intéressent principalement à la théorie arithmétique des formes en lien avec les fonctions elliptiques.

On peut distinguer des approches analytiques et algébriques de la théorie des nombres. Ainsi, l'analyse est centrale dans un ensemble de recherches sur la théorie des nombres premiers - c'est le cas de travaux dans la lignée de Dirichlet ou de Riemann. D’autres auteurs prônent au contraire une approche algébrique de la théorie des nombres. Ici, le sens du mot " algébrique " est multiple: chez Lucas par exemple, l'algèbre renvoie à la théorie des équations tandis que chez Hilbert, l'algèbre renvoie à la théorie des groupes. Finalement, à la fin du siècle, en se fondant sur plusieurs classifications ${ }^{8}$, on retrouve une nouvelle organisation de la théorie des nombres: la théorie des nombres dite élémentaire (congruences, loi de réciprocité quadratique, formes quadratiques dans un certain sens), la théorie des formes, la théorie des corps de nombres algébriques et la théorie des nombres analytique.

Comment situer Catalan dans ce siècle arithmétique? Le premier article de théorie des nombres qu'il publie a pour titre Analyse indéterminée du premier degré et paraît dans Le Géomètre [Catalan 1836], un journal de préparation aux concours des "écoles du gouvernement " qui ne tient que quelques mois en 1836 [Verdier 2009a, p. 82-95]. Dès 1837, ses relations avec Liouville lui permettent de publier ses travaux dans le Journal de mathématiques pures et appliquées: une Note sur la théorie des nombres, portant sur la fonction d'Euler, y est insérée en 1839 [Catalan 1839]. C'est également à cette période que Catalan publie ses premiers textes de combinatoire. Après son élection en 1840 à la Société philomatique, Catalan publie plusieurs papiers dans le Bulletin associé, dont un sur les fractions continues périodiques en 1844 [Catalan 1844b]. Catalan diffuse également ses travaux dans les Nouvelles annales de mathématiques, et ce, dès la création de ce journal mathématique intermédiaire. Là encore, ses relations avec un des éditeurs, Olry Terquem', lui permettent de publier ses articles, de réagir très rapidement aux recherches d'autres auteurs. Ces quelques données montrent que Catalan s'est rapidement forgé une place de choix dans l'espace éditorial français. Notons également qu'à cette période, Catalan se construit progressivement un important réseau relationnel: à côté de Liouville et Terquem, Catalan tisse également des liens - plus ou moins lâches - avec Lamé, Cauchy, Jacobi, Hermite ou encore Tchebychev, qui consacrent tous une partie plus ou moins importante de leurs recherches à la théorie des nombres. Après les années 1840, les travaux de Catalan s'orientent essentiellement et massivement vers ce qui est classé, à la fin du siècle, dans la catégorie "théorie des nombres élémentaire " ${ }^{10}$; il s'agit sur les sommes de diviseurs, sur les équations diophantiennes, sommes de carrés et de cubes, divisibilité des coefficients binomiaux, nombres polygonaux, nombre de solutions d'équations diophantiennes, répartition des nombres premiers, partition d'un nombre, nombres parfaits, etc.

\footnotetext{
${ }^{8}$ Nous nous appuyons ici sur les classifications issues de trois entreprises éditoriales: le Jahrbuch, que nous avons évoqué précédemment; l'Encyklopädie der mathematischen Wissenschaften mit Einschluss ihre Anwendungen (Encyclopédie des sciences mathématiques incluant leurs applications) qui est rédigée sous l'impulsion de Felix Klein et Wilhelm Meyer et qui paraît de 1898 à 1904 [Grattan-Guinness 2009, p. 44-45, 90]; le traité de théorie des nombres de Paul Bachmann, publié en cinq parties entre 1872 et 1905 , dans lesquels il fait une synthèse des différents résultats arithmétiques alors connus, tenant compte de la théorie des nombres élémentaire (Niedere) et avancée (Höhere).

${ }^{9}$ Les deux hommes entament un échange épistolaire régulier en 1839 et le poursuivront jusqu'à la mort de Terquem en 1862.

10 À deux ou trois exceptions près : Catalan sort du cadre " élémentaire " et utilise alors les fonctions elliptiques pour travailler sur les sommes de carrés, en lien avec les travaux de Hermite.
} 


\section{Les débuts arithmétiques de Catalan dans les années 1840}

Comme nous l'avons déjà évoqué, Catalan apparaît comme un des principaux auteurs des deux périodiques français non institutionnels. Du point de vue de l'arithmétique, Catalan publie une Note sur la théorie des nombres dans le Journal de Liouville, qui porte sur la fonction d'Euler ${ }^{11}$, et plusieurs articles dans les Nouvelles annales de mathématiques, dans lesquels il aborde l'analyse indéterminée du premier degré, les fractions continues et les fractions décimales périodiques. Notons que ces trois thématiques appartiennent aux programmes officiels en vigueur, ce qui conforte l'objectif assigné aux Nouvelles annales. Arrêtonsnous cependant sur l'article de Catalan intitulé Sur les fractions décimales périodiques [Catalan 1842] publié dans le premier volume des Nouvelles annales ${ }^{12}$. La présentation choisie par Catalan est intéressante en ce qu'elle s'appuie sur des résultats fondamentaux de la théorie des nombres, la plupart du temps absents des manuels. Catalan revendique d'ailleurs dès son introduction l'originalité de son approche par rapport au contenu traditionnel des manuels :

"La note suivante ne contient rien de neuf : si je me décide à la publier, c'est parce que la manière dont on présente ordinairement la théorie des fractions périodiques n'est, si je ne me trompe, ni très-logique, ni très-rigoureuse. En outre, cette théorie s'appuie assez naturellement sur le théorème de Fermat, et sur d'autres propriétés intéressantes, qu'il serait peut-être convenable de faire entrer dans les éléments. " [Catalan 1842, p. 457].

Dans la suite de son texte, Catalan développe des raisonnements basés sur la considération de restes et obtient des résultats à partir du théorème de Fermat ${ }^{13}$. Il n'emploie à aucun moment le symbole des congruences de Gauss, mais manie à plusieurs reprises la notation " M. 13 " pour désigner un multiple quelconque de 13. Ce mémoire est suivi d'une observation de Terquem, éditeur des Nouvelles annales, dans laquelle il rappelle la définition d'une racine primitive, objet là encore représentatif de la théorie des nombres "à la Gauss ». Il en profite pour introduire une nouvelle notation, différente de celle de Gauss et de celle utilisée ici par Catalan: Terquem utilise le signe $\dot{p}$ pour figurer un multiple de $p$.

Ce texte permet d'éclairer plusieurs aspects de la place de la théorie des nombres dans la communauté enseignante des années 1840 et des méthodes d'échanges au sein d'un périodique intermédiaire comme les Nouvelles annales. Tout d'abord, le texte de Catalan sera cité à plusieurs reprises par différents auteurs du journal: c'est par exemple le cas de Terquem, Eugène Prouhet, Drot ou encore Thibault entre 1842 et 1845. Cela illustre très bien le mode de fonctionnement de ce périodique, dans lequel le lecteur peut suivre de nombreuses discussions déployées sur plusieurs volumes. Ensuite, l'intervention de Terquem en fin de mémoire est tout à fait habituelle: comme Gergonne au début du siècle, Terquem commente très souvent les textes insérés dans "son " journal. Dans le cas de la théorie des nombres, Terquem semble jouer un rôle fondamental dans la mise en avant de l'importance de la théorie des résidus et des congruences. Le cas de Catalan n'est en effet qu'un exemple parmi d'autres. Terquem justifie l'insertion d'un article d'Étienne Midy ainsi: "Cette méthode est précisément celle des congruences de M. Gauss. C'est pour la propager que nous avons inséré cet article " [Midy 1845, p. 147]. Il critique également régulièrement des manuels d'arithmétique qui, selon lui, ne présentent pas une approche adéquate. Dans le cas des Leçons d'arithmétique de P.-L. Cirodde, il observe:

"La divisibilité des nombres, les théorèmes sur les nombres premiers, la recherche des diviseurs communs, précèdent les fractions (33 à 56). Cet ordre est-il bien adapté à l'état actuel de la science telle que nous le devons aux découvertes de Gauss? Il serait plus instructif et en même temps plus facile, de débuter par la théorie des restes, autrement dit des congruences. " [Terquem 1842, p. 54].

Plus généralement, on retrouve dans les années 1840 un réseau d'auteurs des Nouvelles annales,

\footnotetext{
${ }^{11}$ La fonction d'Euler est une fonction arithmétique associant à un nombre entier $n$ le nombre d'entiers naturels inférieurs ou égaux à $n$ et premiers à $n$.

${ }^{12}$ Il est à noter que le premier article des Nouvelles annales porte aussi sur les fractions continues; il s'agit d'un article rédigé par l'autre co-fondateur des Nouvelles annales: Camille Gérono. L'article est de nature historique et pédagogique et ambitionne d'expliquer aux lecteurs de la revue les résultats d'algèbre élémentaire obtenus à l'aide du concept de fraction continue [Gérono 1842].

${ }^{13}$ D'après ce théorème de Fermat, si $p$ est un nombre premier qui ne divise pas un entier $n$, alors $n^{p-1}-1$ est divisible par $p$.
} 
pour la plupart enseignants en collège, dont l'objectif explicité est justement de "propager " une théorie des nombres centrée sur les résidus et les congruences. Terquem y joue le rôle d'animateur, en développant une rhétorique clairement en faveur de cette théorie des nombres et de son intégration dans l'enseignement. Cela contraste bien entendu avec ce que l'on peut trouver dans les media officiels (programmes et manuels). Le rôle de Catalan, s'il n'est pas essentiel dans ce premier cas, nous semble beaucoup plus visible lorsqu'il deviendra lui-même éditeur d'un périodique mathématique intermédiaire, une trentaine d'années plus tard.

\section{Catalan et la Nouvelle correspondance mathématique (NCM) dans les années 1870}

En 1863, Catalan accepte une chaire à l'Université de Liège, en remplacement de Mathias Schaar et se consacre à la préparation des cours dont il est en charge. Il ne cesse pas pour autant de publier dans différentes colonnes: des périodiques français, belges, mais aussi italiens. Il profite également de sa position de membre associé à l'Académie de Belgique pour publier dans les publications de cette institution. Il poursuit également ses relations épistolaires et débute des correspondances avec plusieurs savants belges. On trouve parmi ces deniers Paul Mansion, basé à Gand, et pour qui Catalan joue un rôle fondamental dans la carrière universitaire. Mansion propose en 1870 à Catalan un projet de création de revue mathématique: le premier volume de la Nouvelle correspondance mathématique, édité par les deux hommes, paraît en 1875. Ce journal se propose de traiter principalement des " parties de la science mathématique enseignées " et est composé, comme la plupart des journaux intermédiaires, d'articles originaux, de questions / réponses et d'une partie bibliographique [Voir chapitre 2].

Dans cette période succédant au conflit franco-prussien, cette nouvelle revue mathématique peut être vue comme une "zone neutre " pour les savants français et allemands pendant ses sept années d'existence. Le mathématicien Édouard Lucas par exemple s'en saisit pour diffuser plus largement ses recherches arithmétiques [Décaillot 1999].

Les tables des matières des six volumes parus débutent toutes par la rubrique intitulée « Arithmétique; analyse indéterminée ». Une place importante, en termes de nombres d'articles, est octroyée à cette section et est animé par un réseau d'auteurs international (même s'il est très francophone), lié d'une façon ou d'une autre à Catalan. Catalan intervient sous plusieurs formes (questions, extraits de correspondances, notes sur des mémoires et articles). Ses interventions engendrent des discussions (comme celle sur un ancien mémoire de Libri [Libri 1838]) ou produisent des articles qui sont ensuite repris dans d'autres publications (comme ce problème de Catalan repris par Laisant, cf. partie suivante). En 1879, c'est l'arrêt de la Nouvelle correspondance mathématique; Mansion relance dans la foulée un périodique ayant une forme similaire: Mathesis. Catalan y intervient de manière intensive en posant de nombreuses questions, participant ainsi à la dynamique des auteurs de ce nouveau périodique autour des problèmes arithmétiques.

\section{Itinéraire d'un problème de Catalan ou la théorie des nombres au sein de l'Association française pour l'avancement des sciences (AFAS)}

1872 est l'année de création de trois sociétés savantes en France: la Société mathématique de France (SMF) - la première à être réservée exclusivement aux mathématiques -, la Société française de physique, et l'Association française pour l'avancement des sciences. Dans un contexte politique particulier, juste après la fin du conflit franco-prussien, la fondation de ces sociétés traduit une volonté forte de rétablissement national de la science et de l'enseignement supérieur et de promotion de la recherche scientifique française. Une caractéristique de l'AFAS est de mettre l'accent sur la nécessité d'une alliance entre les milieux politiques, scientifiques et industriels. En mathématiques notamment, cela induit des thèmes de recherche spécifiquement développés dans le cadre des congrès annuels de l'AFAS, qui rassemblent une communauté internationale de scientifiques, dont les Allemands sont cependant exclus jusqu'en 1894 [Décaillot 2002 , Décaillot 2007]. Plusieurs mathématiciens membres de l'AFAS produisent de nombreux travaux de théorie des nombres: c'est par exemple le cas de Laisant ou de Lucas. Catalan, qui est membre de la SMF et de l'AFAS, est le seul mathématicien reconnu à participer au premier congrès de l'AFAS en 1872. Il expose alors un Résumé de sa théorie des polyèdres. Il participe en tout à huit congrès de l'AFAS entre 1872 et 1884 ; il y présente plusieurs travaux arithmétiques, portant sur la somme des diviseurs d'un nombre, sur la répartition des nombres premiers ou encore sur des équations indéterminées. 
Son nom apparaît également dans plusieurs mémoires lus lors de congrès de l'AFAS. C'est notamment le cas d'un mémoire de Laisant [Auvinet 2011, p. 326-340], intitulé Sur le développement de certains produits algébriques [Laisant 1882] qui reprend une question posée par Catalan dans le dernier volume de la Nouvelle correspondance mathématique: "Dans le développement du produit (1-a) (1-b) (1-c) (1-d)... [...], quel est le signe du nième terme?" [Nouvelle correspondance mathématique, vol. 6, p. 143]. Cette question a été résolue dans le même volume par Césaro par une méthode purement combinatoire.

Laisant, à partir du problème posé par Catalan, propose dans un premier temps plusieurs solutions, fondées sur des systèmes de représentations différents. Ainsi, il commence par reprendre la solution présentée par Césaro, puis traduit le problème de Catalan en la considération des permutations possibles dans un alphabet à deux lettres. Une troisième représentation du problème de Catalan s'appuie sur des échiquiers dont les cases sont colorées en noir et blanc. Catalan généralise ensuite le problème en travaillant avec un alphabet à trois lettres, et des échiquiers dont les cases sont de trois couleurs. Cette manière de traiter le problème initial de Catalan renvoie à un courant de recherche plus large et qui semble se développer particulièrement au sein de l'AFAS: en effet, les échiquiers constituent un objet d'étude pour plusieurs mathématiciens, notamment depuis les années 1870 (nous pensons par exemple à Sylvester ou Lucas). De nombreux travaux de ces auteurs s'appuient sur des liens entre théorie des nombres, combinatoire et géométrie de situation ${ }^{14}$. Les mathématiques qu'ils pratiquent correspondent bien à ce qui est attendu au sein de l'AFAS, à savoir une science utile et visuelle. Un acteur important de cette " communauté des mathématiques discrètes " [Auvinet 2011, p. 299-401] est Édouard Lucas. À ce titre, il est très intéressant d'analyser le plan de son ouvrage Théorie des nombres, qui paraît en 1891, qui contient deux chapitres sur l'analyse combinatoire et sur la géométrie de situation, dans lesquels les travaux de Catalan sont cités.

Ces quelques instantanés en lien avec l'activité autour de la théorie des nombres de Catalan, loin de donner une vision exhaustive de ses travaux, permettent néanmoins de saisir quelques facettes du Catalan arithméticien. En tant qu'auteur et rédacteur, Catalan anime plusieurs réseaux d'auteurs particulièrement liés à l'enseignement formés via des journaux intermédiaires: il propose ainsi des exposés sur des thèmes classiques de l'enseignement - comme les fractions décimales périodiques par exemple - selon une nouvelle approche, il commente des articles d'autres auteurs, pose de nombreuses questions arithmétiques initiant ainsi des échanges, ou publie des extraits de correspondances. Il se forge d'ailleurs progressivement l'image d'un arithméticien de valeur: en effet, en 1883, Catalan fait partie de la commission chargée d'examiner les travaux sur le dernier théorème de Fermat dans le cadre d'un prix proposé par l'Académie des sciences de Belgique. Notons enfin que, sans qu'il ne se situe explicitement lui-même dans cette lignée de recherche, certaines de ces thématiques de prédilection - à savoir la combinatoire, la théorie des polyèdres et la théorie des nombres - s'accordent bien avec une approche des mathématiques prônée par plusieurs auteurs de la fin du XIX ${ }^{\mathrm{e}}$ siècle, dont une des figures de proue est Édouard Lucas.

\footnotetext{
${ }^{14}$ Voici une définition de la géométrie de situation par Laisant: "Elle se rattache à la Géométrie par son titre, à l'analyse des combinaisons, et par conséquent à l'Algèbre, par une grande partie des objets qu'elle étudie, à l'Arithmologie par plusieurs de ses conséquences. Enfin, elle rend des services importants dans la Théorie des fonctions et dans les applications géométriques du Calcul infinitésimal. [...] C'est une sorte de Protée affectant des formes diverses, suivant le but poursuivi. » [Laisant 1898, p. 105].
} 


\section{BIBLIOGRAPHIE}

\section{SOURCES PRIMAIRES}

CATALAN (Eugène-Charles)

[1836] " Analyse indéterminée du premier degré ", Le Géomètre, p. 177-180.

[1838] « Note sur un problème de combinaison ", Journal de mathématiques pures et appliquées, vol. 3, p. 111-112.

[1839] « Note sur la théorie des nombres ", Journal de mathématiques pures et appliquées, vol. 4, p. 7-8. [1842] « Sur les fractions décimales périodiques ", Nouvelles annales de mathématiques, I, 1 (1842) p. 457-470. [1844a] " Note extraite d'une lettre adressée à l'éditeur ", Journal für die reine und angewandte Mathematik, vol. 27, p. 192.

[1844b] " Théorèmes sur les fractions continues périodiques simples ", Société Philomatique de Paris. Extraits des procès-verbaux des séances pendant l'année 1844, Paris: Cosson, p. 79-80.

[1888] Mélanges mathématiques. Tome troisième, Bruxelles: F. Hayez.

[1892] « Diverses notes arithmétiques ", Association française pour l'avancement des sciences. Compte rendu de la $20^{e}$ session. Marseille 1891, Paris: Masson, p. 194-204.

GAUSS (Carl Friedrich)

[1801] Disquisitiones Arithmeticae, Leipzig: Fleischer.

GÉRONO (Camille)

[1842] « Fraction continue », Nouvelles annales de mathématiques, I, 1 (1842) p. 1-20.

LAISANT (Charles-Ange)

[1882] "Sur le développement de certains produits algébriques ", Association française pour l'avancement des sciences. Compte rendu de la 10 session. Alger 1881, Paris: Masson, p. 84-108.

[1898] La mathématique: philosophie-enseignement, Paris: G. Carré et C. Naud.

LEGENDRE (Adrien-Marie)

[1798] Essai sur la théorie des nombres, Paris: Duprat.

LIBRI (Guglielmo)

[1838] " Mémoire sur la théorie des nombres ", Mémoires présentés par divers Savants à l'Académie Royale des Sciences de l'Institut de France; sciences mathématiques et physiques, vol. 5, p. 1-75.

MIDY (Étienne)

[1845] « Analyse indéterminée du premier degré », Nouvelles annales de mathématiques, I, 4 (1845) p. 146-152.

TERQUEM (Olry)

[1842] « Analyse d'ouvrages nouveaux ", Nouvelles annales de mathématiques, I, 1 (1842) p. 49-56. 


\section{SOURCES SECONDAIRES}

AUVINET (Jérôme)

[2011] Charles-Ange Laisant. Itinéraires et engagements d'un mathématicien, d'un siècle à l'autre (1841-1920), Thèse de doctorat, Nantes: Université de Nantes.

BELHOSTE (Bruno)

[1995] Les sciences dans l'enseignement secondaire français. Textes officiels. Tome I: 1789-1914, Paris : INRP.

BOUCARD (Jenny)

[2011] Un "rapprochement curieux de l'algèbre et de la théorie des nombres ": études sur l'utilisation des congruences de 1801 à 1850, Thèse de doctorat, Paris: Université Paris 6.

DÉCAILLOT (Anne-Marie)

[1999] Édouard Lucas (1842-1891): le parcours original d'un scientifique français dans la deuxième moitié $d u X I X^{e}$ siècle, Thèse de doctorat, Paris: Université René Descartes, Paris V.

[2002] "Originalité d'une démarche mathématique ", in GISPERT, Hélène (dir.), "Par la science, pour la patrie ". L'Association française pour l'avancement des Sciences (1872-1914), un projet politique pour une société savante, Rennes: Presses Universitaires de Rennes, p. 205-214.

[2007] "Number Theory at the Association française pour l'avancement des sciences ", in [Goldstein \& al. 2007, p. 411-427].

DICKSON (Leonard Eugene)

[1919-1923] History of the Theory of Numbers, Washington: Carnegie Institue of Washington, 3 vols.

GOLDSTEIN (Catherine)

[1999] «Sur la question des méthodes quantitatives en histoire des mathématiques: le cas de la théorie des nombres en France (1870-1914) ", Acta bistoriae rerum naturalium nec non technicarum, vol. 28, p. $187-214$.

GOLDSTEIN (Catherine) \& SCHAPPACHER (Norbert)

[2007a] "A Book in Search of a Discipline (1801-1860) ", in [Goldstein \& al. 2007, p. 3-65].

[2007b] "Several Disciplines and a Book (1860-1901)", in [Goldstein \& al. 2007, p. 67-103].

GOLDSTEIN (Catherine), SCHAPPACHER (Norbert) \& SCHWERMER (Joachim)

[2007] The Shaping of Arithmetic after C. F. Gauss's Disquisitiones Arithmeticae, Berlin: Springer.

GRATTAN-GUINNESS (Ivor)

[2009] Routes of learning: highways, pathways, and byways in the bistory of mathematics, Baltimore: Johns Hopkins University Press.

JONGMANS (François)

[1996] Eugène Catalan: géomètre sans patrie, républicain sans république, Mons: Société belge des professeurs de mathématique d'expression française.

\section{VERDIER (Norbert)}

[2009a] Le Journal de Liouville et la presse de son temps: une entreprise d'édition et de circulation des mathématiques au XIX ${ }^{e}$ siècle (1824-1885), Thèse de doctorat, Paris: Université Paris-Sud.

[2009b] "Les journaux de mathématiques dans la première moitié du XIX siècle en Europe ", Philosophia Scientice, vol. 13, n 2, p. 97- 126. 